\title{
A Drug Safety Rating System Based on Postmarketing Costs Associated with Adverse Events and Patient Outcomes
}

\author{
Keith B. Hoffman, PhD; Mo Dimbil, BS; Robert F. Kyle, JD; Nicholas P. Tatonetti, PhD;
} Colin B. Erdman, BA; Andrea Demakas, BA; Dingguo Chen, BS; and Brian M. Overstreet, BA

\begin{abstract}
BACKGROUND: Given the multiple limitations associated with relatively homogeneous preapproval clinical trials, inadequate data disclosures, slow reaction times from regulatory bodies, and deep-rooted bias against disclosing and publishing negative results, there is an acute need for the development of analytics that reflect drug safety in heterogeneous, real-world populations.
\end{abstract}

OBJECTIVE: To develop a drug safety statistic that estimates downstream medical costs associated with serious adverse events (AEs) and unfavorable patient outcomes associated with the use of 706 FDA-approved drugs.

METHODS: All primary suspect case reports for each drug were collected from the FDA's Adverse Event Reporting System database (FAERS) from 2010-2014. The Medical Dictionary for Regulatory Activities (MedDRA) was used to code serious AEs and outcomes, which were tallied for each case report. Medical costs associated with AEs and poor patient outcomes were derived from Agency for Healthcare Research and Quality (AHRQ) survey data, and their corresponding ICD-9-CM codes were mapped to MedDRA terms. Nonserious $A E s$ and outcomes were not included. For each case report, either the highest $A E$ cost or, if no eligible $A E$ was listed, the highest outcome cost was used. All costed cases were aggregated for each drug and divided by the number of patients exposed to obtain a downstream estimated direct medical cost burden per exposure. Each drug was assigned a corresponding 1-100 point total.

RESULTS: The 706 drugs showed an exponential distribution of downstream costs, and the data were transformed using the natural log to approximate a normal distribution. The minimum score was 8.29 , and the maximum score was 99.25 , with a mean of 44.32 . Drugs with the highest individual scores tended to be kinase inhibitors, thalidomide analogs, and endothelin receptor antagonists. When scores were analyzed across Established Pharmacologic Class (EPC), the kinase inhibitor and endothelin receptor antagonist classes had the highest total. However, other EPCs with median scores of 75 and above included hepatitis $C$ virus NS3/4A protease inhibitor, recombinant human interferon beta, vascular endothelial growth factor-directed antibody, and tumor necrosis factor blocker. When Anatomical Therapeutic Chemical classifications were analyzed, antineoplastic drugs were outliers with approximately $80 \%$ of their individual scores 60 and above, while approximately $20 \%-30 \%$ of blood and antiinfective drugs had scores of 60 and above. Within-drug class results served to differentiate similar drugs. For example, 6 serotonin reuptake inhibitors had a score range of 35 to 53 .

CONCLUSIONS: This scoring system is based on estimated direct medical costs associated with postmarketing AEs and poor patient outcomes and thereby helps fill a large information gap regarding drug safety in real-world patient populations.

J Manag Care Spec Pharm. 2015;21(12):1134-43

Copyright $\odot 2015$, Academy of Managed Care Pharmacy. All rights reserved.

\section{What is already known about this subject}

Preapproval clinical trials cannot predict many adverse drug reactions that are observed in real-world patient populations.

Postmarketing adverse event reporting has increased dramatically over the past decade, with approximately 1,500,000 reports now being submitted to the FDA's Adverse Event Database (FAERS) annually.

FAERS data are not widely used by health care decision makers.

\section{What this study adds}

Postmarketing adverse event reports can be assigned direct medical costs in order to estimate a drug's downstream financial impact.

By quantifying reported adverse drug events in the postapproval phase of a drug into downstream direct medical costs, this system can serve as a needed window into drug safety in real-world patient populations

Adverse events and patient outcome data were obtained from FAERS, and direct medical costs were estimated for 706 FDAapproved drugs.

T n order to increase the likelihood that drug efficacy signals can be detected during clinical trials, pharmaceutical developers purposefully enroll subjects who are relatively homogenous. This procedural step, while vital for achieving robust statistical descriptions of a compound's efficacy, necessarily leaves open the possibility that the test agent will have unexpected actions once it is used in a heterogeneous population of patients.

Often, serious and life-threatening side effects that were not exposed during preapproval screening programs become evident only after drug approval. A member of the U.S. Food and Drug Administration's (FDA) Office of Drug Safety summed up the issue by stating, "The complete adverse event profile of a drug is not known at the time of approval because of the small sample size, short duration, and limited generalizability of pre-approval clinical trials." Also, "since most trials exclude the elderly, children, pregnant women, patients with multiple diseases, and those on medications suspected of interaction with the study drug, the studies' participants may not be representative of the real world where the drug is eventually used." 
The gradual evolution of side-effect profiles across numerous drug classes only after they win FDA approval serves to underscore the preceding points (examples include severe cardiac complications from the weight management drug Meridia, ${ }^{2}$ a fatal muscle-wasting syndrome from the cholesterol management drug Baycol, ${ }^{3}$ and increased heart attack and stroke rates in patients taking Vioxx, prescribed for osteoarthritis and joint pain $^{4}$ ). In short, careful postapproval monitoring is vital to the ongoing drug evaluation process.

Physicians and pharmacists routinely obtain safety information from drug label "inserts" that are often based predominately on preapproval clinical trial results. It is this reliance on incomplete safety data derived from limited clinical trial systems that can contribute to a significant gap in knowledge for practicing health care providers., ${ }^{1,5,6}$

The FDA's Adverse Event Reporting System (FAERS) is a centralized computerized information database that is used for postmarketing drug safety surveillance. FAERS is currently growing by approximately 1,500,000 new cases per year. ${ }^{7}$ FDA professionals and pharmacovigilance experts routinely look to FAERS data as a guide to, and signal generator of, drug safety issues. Both groups employ a wide array of sophisticated data mining and signal detection techniques (for reviews, see Tatonetti et al., 2012, and Harpaz et al., 2012 $2^{8,9}$ ). The FDA uses such analyses to issue warnings, mandate label changes, and remove drugs from the U.S. market after an incidence, or severity, of their side effects is determined to significantly differ from what preapproval clinical trial results previously suggested. ${ }^{10}$ FAERS, and other similar spontaneous reporting systems maintained by governmental and international organizations, are a main resource for identifying postmarketing drug safety concerns. ${ }^{1,11-25}$

Detailed FAERS data, however, are neither readily accessible nor organized in a way that is useful to broad health care audiences. We believe that the data contained in FAERS represent valuable insight into real-world drug safety risks. Accordingly, we built the drug scoring system described in this article to provide a readily accessible evaluation of a drug's potential safety risks. The system is based on serious adverse events (AEs) and patient outcomes listed in postmarketing case reports. It assesses drug safety by estimating the magnitude of downstream direct medical costs based on AE and outcome costing data taken from the Agency for Healthcare Research and Quality's (AHRQ) Healthcare Cost and Utilization Project (HCUP). ${ }^{26,27}$

\section{Methods}

\section{Adverse Event and Outcome Data}

$\mathrm{AE}$ and patient outcome data were obtained for 706 FDAapproved drugs during the time period of January 2010 through December 2014 from an analytic system of FAERS case reports. ${ }^{28}$ Nonserious and disease-related AEs were ignored, as well as nonserious outcomes.

\section{FAERS Case Reports}

To import and filter data from FAERS, common data preprocessing techniques were used to normalize and qualify textual data, such as removal of nonalphanumeric characters, white spaces, and line breaks. Filtering processes included the following: (a) a system for automated name matching that corrected for drug name misspellings and incorrect data within major fields (i.e., the inclusion of dosages or routes of administration as part of the drug name field); (b) aggregation of generic and non-U.S. brand name drugs under a single brand name; (c) separation of "primary suspect" and "all suspect" designations; and (d) identification of common AE and condition types. Automated data preprocessing and scrubbing workflow provided an initial assignment of "raw" FAERS drug names. The automated matching process utilized a combination of fuzzy string matching, string distance, and phonetic matching algorithms.

\section{Inclusion Criteria for FAERS Case Reports}

Case reports that were missing or contained malformed key identification fields (Individual Safety Report number [ISR], patient number, drug sequence identification, or AE terms from the Medical Dictionary for Regulatory Activities [MedDRA] ${ }^{29}$ ) were discarded. As long as the previously mentioned key identification fields were contained in a given case report, allowable missing fields included age, gender, weight, outcome, and condition. Cases were discarded if the drug name was found to be indeterminate or if the name was determined to not represent an FDA-approved drug (e.g., dietary supplements or foods). In instances where there was more than 1 Individual Case Safety Report (ICSR) for the same identification number in the same calendar year, the earliest reported case was selected (Appendix A, available in online article).

\section{Drug Name Mapping and Established Pharmacologic Classes}

Drug name text-mapping was accomplished as described by Hoffman et al. (2013). ${ }^{28}$ Drug names were normalized to RxNorm reference codes using string searching and manual curation..$^{30}$ National Drug File Reference Terminology was used to provide ancillary information on class and mechanism of action. $^{31}$

Established Pharmacological Class (EPC) is a designation found in the FDA's National Drug Code file that indicates an established pharmacologic class, as required by the FDA's structured product labeling process. ${ }^{32}$ All drugs were sorted into their corresponding EPCs. Score averages were calculated for each EPC.

\section{Adverse Event Coding}

AE information was coded according to MedDRA version 17.1. ${ }^{29}$ "Primary suspect" designations in FAERS case reports were quantified in an attempt to restrict the analysis to those drugs directly suspected of causing the AE. A discrepancy 


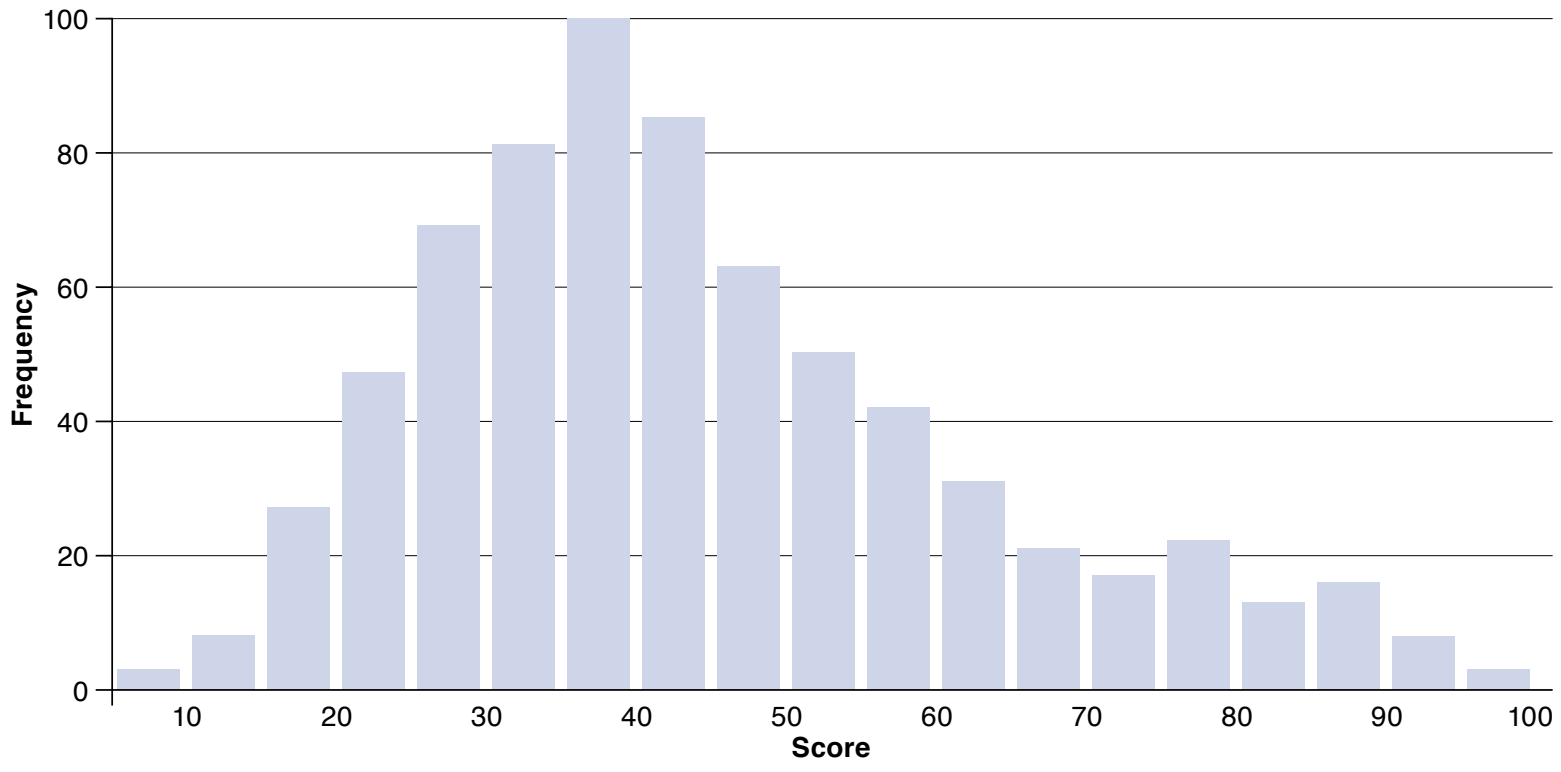

occasionally observed with FAERS case reports is that diseaserelated symptoms are sometimes listed in the "adverse event" field. In instances where such mistakes were easily identifiable, we excluded those "AEs" from analysis. For example, approximately $1 \%$ of the case reports for pramipexole dihydrochloride and approximately $1 \%$ of the case reports for donepezil hydrochloride listed Parkinson's disease and dementia, respectively, as an AE. Such case reports are not included in our analyses.

\section{Costs and ICD-9-CM Mapping}

HCUP is a compilation of patient data collected by the AHRQ, that is coded to the International Classification of Diseases, Ninth Revision, Clinical Modification (ICD-9-CM). ${ }^{26,27}$ We used HCUP to obtain national hospitalization and aggregate costs for specific diagnoses and procedures.

Given that the FAERS database is MedDRA coded, we used BioPortal, a repository of biomedical ontologies, and an ICD-9-CM mapping resource to assign ICD-9-CM codes to MedDRA Preferred Term (PT) AEs. ${ }^{33,34}$ For case reports in which no eligible direct medical cost was assigned, we used AHRQ "outcome" figures. ${ }^{35}$ We were able to assign costs to 1,508 serious MedDRA PT AEs. Of those PT AEs, 1,213 (80\%) were mapped using BioPortal to ICD-9-CM codes with available cost data; 295 PT AEs (20\%) that could not be assigned by the use of BioPortal were manually mapped using the ICD-9-CM coding manual with the following hierarchy ${ }^{34}$ : (a) verbatim match (e.g., MedDRA PT "asphyxia" was mapped to ICD-9-CM code 799.01, asphyxia); (b) PTs matched to broader ICD-9-CM categories (e.g., MedDRA PT "nephrogenic systemic fibrosis" was mapped to ICD-9-CM code 710.8, other specified diffuse diseases of connective tissue); and (c) for terms that were mapped to multiple ICD-9-CM categories, we obtained a weighted average of the relevant direct medical cost data (e.g., MedDRA PT "cardio-respiratory arrest" was mapped to ICD-9-CM codes 427.5, cardiac arrest, and 799.1, respiratory arrest). Appendix B (available in online article) shows examples of costs and ICD-9-CM mapping.

We limited our focus to only those terms included in the EudraVigilance Important Medical Event Terms list. ${ }^{36}$

\section{Primary Suspect Case Reports and Incidence Data}

For each drug we selected, all reported primary suspect AEs from January 2010 through December 2014 were obtained. In cases with more than 1 eligible $\mathrm{AE}$, only the $\mathrm{AE}$ with the largest individual cost was selected for each case. For example, it was sometimes observed that similar AEs were listed in a single case report (e.g., cerebral hemorrhage at $\$ 21,273$ and ischaemic stroke at $\$ 14,858$, or pulmonary embolism at $\$ 14$, 878 and pulmonary infarction at $\$ 10,804)$, so we decided a "most costly" AE selection would better align with actual medical expenditures. In cases with no eligible $\mathrm{AE}$, but with a listed outcome, we selected only the largest outcome direct medical cost per case. We divided the total direct medical costs derived from a drug's case reports by the number of patients exposed over the same time period to obtain a direct medical cost per drug. Patient usage data for 2010, 2011, and 2012 were based on information derived from the Medical Expenditure Panel Survey (MEPS). ${ }^{37}$ Given that MEPS is only available through 
TABLE 1 The 50 Highest Scores, Corresponding EPC, and Number of Case Reports Analyzed

\begin{tabular}{|c|c|c|c|}
\hline Compound & Score & EPC & Number of Case Reports \\
\hline Pomalidomide & 99.25 & Thalidomide analog & 2,755 \\
\hline Lenalidomide & 96.72 & Thalidomide analog & 23,591 \\
\hline Ruxolitinib & 95.58 & Kinase inhibitor & 2,230 \\
\hline Bosentan & 94.97 & Endothelin receptor antagonist & 17,346 \\
\hline Ambrisentan & 93.97 & Endothelin receptor antagonist & 14,542 \\
\hline Pazopanib & 93.66 & Kinase inhibitor & 2,790 \\
\hline Everolimus & 91.94 & Kinase inhibitor & 4,881 \\
\hline Ibrutinib & 91.88 & Kinase inhibitor & 911 \\
\hline Deferasirox & 91.11 & Iron chelator & 6,945 \\
\hline Regorafenib & 91.06 & Kinase inhibitor & 856 \\
\hline Dabrafenib & 90.96 & Kinase inhibitor & 284 \\
\hline Carfilzomib & 89.97 & Proteasome inhibitor & 559 \\
\hline Brentuximab vedotin & 89.44 & CD30-directed immunoconjugate & 558 \\
\hline Ipilimumab & 89.19 & CTLA-4-directed blocking antibody & 2,217 \\
\hline Macitentan & 89.04 & Endothelin receptor antagonist & 343 \\
\hline Enzalutamide & 88.82 & Androgen receptor inhibitor & 2,363 \\
\hline Peginterferon alfa- $2 \mathrm{a}$ & 88.49 & Interferon alpha & 3,656 \\
\hline Trametinib dimethyl sulfoxide & 88.12 & Kinase inhibitor & 170 \\
\hline Sunitinib malate & 88.04 & Kinase inhibitor & 6,202 \\
\hline Azacitidine & 87.67 & Nucleoside metabolic inhibitor & 2,391 \\
\hline Obinutuzumab & 87.55 & CD20-directed cytolytic antibody & 304 \\
\hline Sorafenib tosylate & 86.51 & Kinase inhibitor & 4,043 \\
\hline Ivacaftor & 86.00 & Cystic fibrosis transmembrane conductance regulator potentiator & 263 \\
\hline Cetuximab & 85.92 & Epidermal growth factor receptor antagonist & 2,877 \\
\hline Teriflunomide & 85.77 & Pyrimidine synthesis inhibitor & 1,090 \\
\hline Natalizumab & 85.63 & Integrin receptor antagonist & 21,204 \\
\hline Gemcitabine & 85.34 & Nucleoside metabolic inhibitor & 2,376 \\
\hline Zoledronic acid (Reclast) & 84.51 & Bisphosphonate & 2,894 \\
\hline Bevacizumab & 83.85 & Vascular endothelial growth factor-directed antibody & 14,385 \\
\hline Interferon beta-la (Rebif) & 83.82 & Recombinant human interferon beta & 12,474 \\
\hline Telaprevir & 83.57 & Hepatitis C virus NS3/4A protease inhibitor & 7,281 \\
\hline Bendamustine & 83.06 & Alkylating drug & 1,980 \\
\hline Ado-trastuzumab emtansine & 82.99 & HER2-targeted antibody-drug conjugate & 363 \\
\hline Tocilizumab & 82.69 & IL-6 receptor antagonist & 5,284 \\
\hline Lomitapide & 82.63 & Microsomal triglyceride transfer protein inhibitor & 39 \\
\hline Erlotinib & 82.49 & Kinase inhibitor & 15,815 \\
\hline Bortezomib & 82.10 & Proteasome inhibitor & 3,984 \\
\hline Sipuleucel-t & 81.81 & Autologous cellular immunotherapy & 778 \\
\hline Teriparatide & 81.10 & Parathyroid hormone analog & 17,124 \\
\hline Rosiglitazone & 80.04 & Peroxisome proliferator receptor gamma agonist; thiazolidinedione & 15,621 \\
\hline Tofacitinib & 79.88 & Kinase inhibitor & 744 \\
\hline Dasatinib & 79.78 & Kinase inhibitor & 1,574 \\
\hline Sodium oxybate & 79.36 & Central nervous system depressant & 2,697 \\
\hline Ramucirumab & 79.32 & Vascular endothelial growth factor-directed antibody & 51 \\
\hline Clozapine (Clozaril) & 79.06 & Atypical antipsychotic & 8,449 \\
\hline Imatinib & 78.71 & Kinase inhibitor & 11,902 \\
\hline Oxaliplatin & 78.64 & Platinum-based drug & 1,594 \\
\hline Fingolimod & 78.34 & Sphingosine 1-phosphate receptor modulator & 4,961 \\
\hline Nilotinib & 78.06 & Kinase inhibitor & 3,353 \\
\hline Dimethyl fumarate & 77.87 & Immunomodulator for RRMSa & 4,361 \\
\hline
\end{tabular}




\section{TABLE 2 The 15 Highest Scoring EPCS \\ Where Each Class Had 3 or \\ More Drug Members and 3,000 or More Case Reports}

\begin{tabular}{l|c|c|c}
\hline EPC & Score & $\begin{array}{c}\text { Number } \\
\text { Costed } \\
\text { Cases }\end{array}$ & $\begin{array}{c}\text { Number } \\
\text { of Drugs } \\
\text { in this } \\
\text { Class }\end{array}$ \\
\hline Endothelin receptor antagonist & 94.39 & 32,231 & 3 \\
\hline Kinase inhibitor & 82.66 & 55,011 & 13 \\
\hline Hepatitis C virus NS3/4A protease inhibitor & 79.52 & 8,140 & 3 \\
\hline Recombinant human interferon beta & 79.29 & 26,095 & 3 \\
\hline Vascular endothelial growth factor-directed & 77.22 & 23,074 & 4 \\
antibody & & & \\
\hline Tumor necrosis factor blocker & 74.74 & 102,779 & 5 \\
\hline Alkylating drug & 72.63 & 3,977 & 3 \\
\hline Microtubule inhibitor & 71.50 & 3,170 & 3 \\
\hline Nucleoside metabolic inhibitor & 66.81 & 8,442 & 6 \\
\hline Factor Xa inhibitor & 62.49 & 12,926 & 3 \\
\hline Thiazolidinedione & 59.16 & 19,079 & 5 \\
\hline Peroxisome proliferator receptor gamma agonist & 59.16 & 19,079 & 5 \\
\hline Calcineurin inhibitor immunosuppressant & 56.36 & 6,383 & 7 \\
\hline GLP-1 receptor agonist & 55.57 & 7,002 & 3 \\
\hline $\begin{array}{l}\text { HIV nucleoside analog reverse transcriptase } \\
\text { inhibitor }\end{array}$ & 54.16 & 4,039 & 13 \\
\hline EPC=Established Pharmacologic Class. & & & \\
\hline
\end{tabular}

2012, we used sales-based figures provided by Evaluate Pharma for 2013 and 2014. These figures are estimates of the number of patients in the U.S. market receiving a drug in a given year. They are calculated based on disclosed U.S. sales divided by the revenues per patient per year (cost per patient, adjusted for patient compliance rate [\%], and off-invoice discounts).

\section{Conversion to 1-100 Scale}

We observed that the minimum direct medical cost per patient exposure was approximately $\$ 0.02$, and the maximum was approximately $\$ 10,000$. These values appeared to be distributed exponentially. Accordingly, the raw cost data were transformed using the natural log to approximate a normal distribution. The minimum log-adjusted direct medical cost per patient was -4 and the maximum was 9.2. To scale those values to form a 1-100 scale, we used the following formula: $(\ln (\mathrm{x})+4) * 7.5$.

\section{Results}

\section{Score Distributions and Top $\mathbf{5 0}$ Highest Scoring Drugs}

The total number of drugs included in this analysis was 706 . The minimum score was 8.29 (cost of \$0.02) and the maximum score was $99.25(\$ 10,220)$. The median, mean, and standard deviation were 40.58 (\$4.10), 44.45 (\$6.87), and 18.29 (\$0.21), respectively. There were 79 drugs with scores of 70 and above (\$207.13), while 131 drugs had scores of 60 and above (\$54.60). Figure 1 shows the distribution of scores across all 706 drugs, and Table 1 shows the top 50 highest scores, corresponding EPC, and the number of case reports analyzed. While the top 50 drugs comprised various drug classes, the following classes had more than 1 drug listed in the top 50: kinase inhibitor (14 individual drugs), endothelin receptor antagonist (3), nucleoside metabolic inhibitor (2), proteasome inhibitor (2), thalidomide analog (2), and vascular endothelial growth factordirected antibody (2).

Factors contributing to high scores were elevated associations with significant AEs. For example, the 2 highest scoring drugs were thalidomide analogs, with pomalidomide having 241 case reports of pneumonia $(\$ 2,099,833$ total; $\$ 8,713$ each); 90 reports of pancytopenia ( $\$ 1,032,840$ total; $\$ 11,476$ each); and 82 reports of neutropenia (\$990,806 total; $\$ 12,083$ each). Lenalidomide had 1,673 case reports of pneumonia ( $\$ 14,576,849$ total; $\$ 8,713$ each); 889 reports of pancytopenia $(\$ 10,202,164$ total; $\$ 11,476$ each); and 811 reports of neutropenia (\$9,799,313 total; $\$ 12,083$ each). For the 2 highest scoring endothelin receptor antagonists, bosentan had 477 case reports of pneumonia $(\$ 4,156,101$ total; $\$ 8,713$ each); 245 reports of congestive cardiac failure ( $\$ 2,492,385$ total; $\$ 10,173$ each); and 231 reports of respiratory failure $(\$ 4,296,369$ total; $\$ 18,599$ each), while ambrisentan had 1,220 case reports of pneumonia ( $\$ 10,629,860$ total; $\$ 8,713$ each); 464 reports of congestive cardiac failure ( $\$ 4,720,272$ total; $\$ 10,173$ each); and 256 reports of respiratory failure $(\$ 4,761,344$ total; $\$ 18,599$ each).

\section{Established Pharmacologic Classes}

EPC is a designation found in the FDA's National Drug Code file that indicates an established pharmacologic class, as required by FDA's structured product labeling requirements. ${ }^{32}$ All drugs were sorted into their corresponding EPC classes. Endothelin receptor antagonists and kinase inhibitors were the 2 EPCs with the highest weighted averages (Table 1). Other EPCs with median scores of 70 and above included hepatitis $C$ virus NS3/4A protease inhibitor, recombinant human interferon beta, vascular endothelial growth factor-directed antibody, tumor necrosis factor blocker, alkylating drug, and microtubule inhibitor. Table 2 shows 15 of the highest scoring EPCs where each class had 3,000 or more cases reports and 3 or more individual drug members.

In an attempt to highlight EPCs that might pose a high level of risk to a large number of patients, Appendix C (available in online article) shows the distribution of weighted average scores for each EPC that comprised 3 or more individual compounds and had 3,000 or more case reports over the time period studied. The tumor necrosis factor blocker EPC was an outlier because of a high number of case reports combined with a high score average. Other EPCs with the combination of a weighted average above 60 and over 20,000 costed cases were vascular endothelial growth factor-directed antibody, recombinant human interferon beta, kinase inhibitor, and endothelin 


\section{FIGURE 2 Individual Drug Scores Mapped to Corresponding ATC Codes}

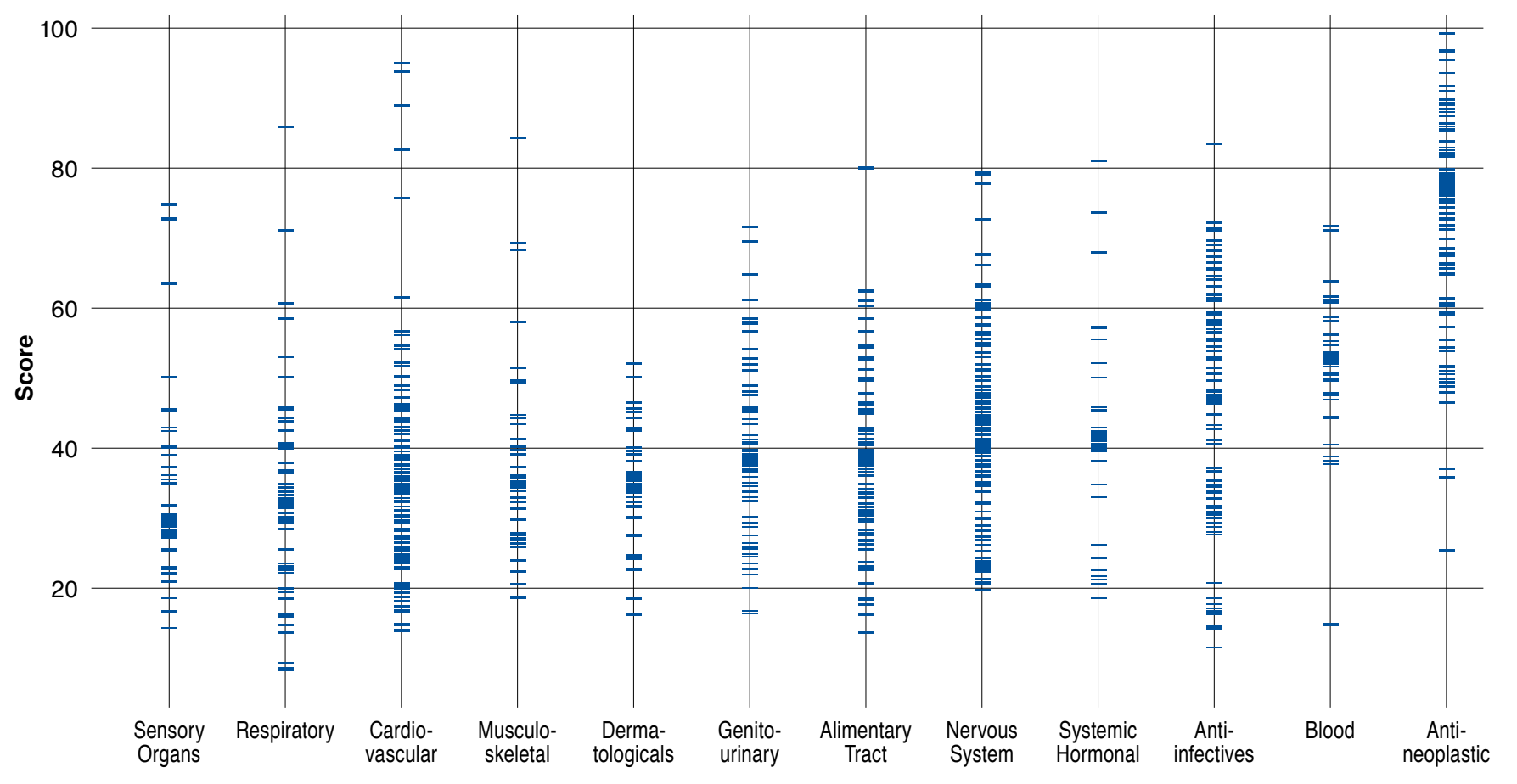

Note: Scores are noted on the y axis. Aggregated ATC groups have their individual scores plotted vertically with a thick band indicating the median of each group. The lowest average group scores are to the left while the highest are to the right.

ATC=Anatomical Therapeutic Chemical.

receptor antagonist, suggesting that they represent elevated risks across large populations of patients. EPCs with lower risk and lower case counts were penicillin-class antibacterial, angiotensin-converting enzyme inhibitor, beta-adrenergic blocker, and thiazide diuretic.

In Figure 2, individual drug scores were mapped to their corresponding Anatomical Therapeutic Chemical (ATC) codes, another widely used drug classification system based on the site of drug action as well as pharmacologic, chemical, and therapeutic properties. ${ }^{38}$ Only ATC groups with 10 or more individual drug members are included in this figure.

To determine which ATC groups were associated with a high percentage of elevated scores, we plotted the percentage of scores that were 60 and above for each group with 10 or more individual drugs. Appendix D (available in online article) shows that antineoplastic drugs were outliers with approximately $80 \%$ of their individual scores at 60 and above. Blood and anti-infective drugs had the second and third highest percentage ( $20 \%-30 \%)$ of scores that were 60 and above. In contrast, respiratory, genitourinary, and cardiovascular groups had the lowest percentage of scores that were 60 and above, respectively.

\section{Specific Drug Classes}

Table 3 shows examples of score ranges within the following specific drug classes: protein kinase inhibitors, serotoninreuptake inhibitors, proton pump inhibitors, and macrolide antimicrobials. Each drug class included in Table 3 had 150 or more costed cases.

\section{Discussion}

Preapproval clinical trial participants are subjected to rigorous inclusion and exclusion criteria in order to increase the likelihood that drug efficacy signals can be detected during clinical testing. Such selection processes are vital for determining a compound's efficacy and are also usually necessary for financial and logistical reasons. The downside of such methods, especially with regard to determining the safety profile of a drug, is that enrolled subjects are relatively homogenous. The preapproval clinical trial process, therefore, often fails to uncover many of the side effects that occur once the drug is introduced to real-world, heterogeneous patient populations. Accordingly, AEs from drugs approved by the FDA are a major public safety concern. In fact, approximately 1,500,000 $\mathrm{AE}$ reports are currently submitted to FAERS, the FDA's 


\begin{tabular}{|c|c|c|}
\hline $\begin{array}{rr}\text { TABLE 3 Exa } & \text { Dru } \\
& 150\end{array}$ & $\begin{array}{l}\text { anges } \\
\text { Comp } \\
\text { es }\end{array}$ & $\begin{array}{l}\text { in Specific } \\
\text { As with }\end{array}$ \\
\hline Compound & Score & $\begin{array}{c}\text { Number of } \\
\text { Costed Cases }\end{array}$ \\
\hline \multicolumn{3}{|l|}{ Protein kinase inhibitors } \\
\hline Ruxolitinib & 95.58 & 2,230 \\
\hline Pazopanib & 93.66 & 2,790 \\
\hline Everolimus & 91.94 & 4,881 \\
\hline Ibrutinib & 91.88 & 911 \\
\hline Regorafenib & 91.06 & 856 \\
\hline Dabrafenib & 90.96 & 284 \\
\hline Sunitinib & 88.04 & 6,202 \\
\hline Sorafenib & 86.51 & 4,043 \\
\hline Erlotinib & 82.49 & 15,815 \\
\hline Tofacitinib & 79.88 & 744 \\
\hline Dasatinib & 79.78 & 1,574 \\
\hline Imatinib & 78.71 & 11,902 \\
\hline Nilotinib & 78.06 & 3,353 \\
\hline \multicolumn{3}{|c|}{ Serotonin-reuptake inhibitors } \\
\hline Paroxetine (Paxil CR) & 53.16 & 215 \\
\hline Paroxetine (Paxil) & 46.21 & 2,803 \\
\hline Fluoxetine & 40.18 & 2,454 \\
\hline Sertraline & 39.85 & 3,273 \\
\hline Escitalopram & 36.62 & 1,745 \\
\hline Citalopram & 35.91 & 2,573 \\
\hline \multicolumn{3}{|l|}{ Proton pump inhibitors } \\
\hline Esomeprazole & 46.56 & 9,344 \\
\hline Esomeprazole, naproxen & 40.28 & 150 \\
\hline Rabeprazole & 36.52 & 402 \\
\hline Lansoprazole & 33.42 & 647 \\
\hline Pantoprazole & 32.08 & 885 \\
\hline Omeprazole & 26.80 & 1,780 \\
\hline \multicolumn{3}{|l|}{ Macrolide antimicrobials } \\
\hline Clarithromycin & 43.36 & 1,200 \\
\hline Erythromycin & 31.72 & 272 \\
\hline Azithromycin & 16.32 & 839 \\
\hline
\end{tabular}

repository of postmarketing AEs each year, and this figure is widely believed to be an underestimation of the actual amount of AEs triggered by approved drugs. ${ }^{\text {? }}$

When performing comparative effectiveness research, health care decision makers routinely obtain safety information from drug label inserts, which are dossiers prepared by pharmaceutical companies, and preapproval clinical trial results. Such data are limited in scope by publication bias, commercial interests, disclosure delays, and the homogeneity of test subjects.

Drug label inserts are largely based on clinical trial data, but such data are associated with serious publication bias. ${ }^{39-45}$ For example, in a large study of over 100 clinical trials, $65 \%$ of harms outcomes were incompletely reported. ${ }^{46}$

Pharmacy and therapeutics (P\&T) committees are subjected to pharmaceutical company bias, since they rely heavily on dossiers prepared by drug manufactures. Tellingly, only $43 \%$ of respondents in a recent Ernst \& Young survey agreed with the statement that "pharmaceutical companies have data that are credible for measuring and improving outcomes." ${ }^{\text {"47 }}$ Additionally, while "curbing rising drug costs" was one of the biggest concerns noted in the same survey, "curbing rising medical (non-drug) costs" was the second biggest payer concern. Finally, boosting drug adherence was noted as a critical component of lowering health care costs. The lack of real-world AE data hampers P\&T decisions aimed at addressing these issues.

Accordingly, there is an obvious gap in drug safety information flow regarding real-world medication use. One way to address the deficiencies associated with the clinical trial system and currently available drug safety information would be to enhance the use of postapproval AE data.

Numerous studies have documented the utility of FAERS for generating safety signals. ${ }^{13,14,17-21,23,24,48-50}$ Accordingly, the benefit of using FAERS data to fill information gaps left by preapproval safety testing appears clear. However, meaningful analysis of FAERS largely depends on expensive, complex, and proprietary data mining and signaling tools. These limitations block routine FAERS data usage by most health care decision makers.

Therefore, in order to make FAERS data accessible and useful for health care decision makers, we developed an analytic to estimate direct medical costs associated with AEs and patient outcomes linked to 706 FDA-approved drugs. Data were derived from postmarketing reports submitted to the FDA rather than from preapproval clinical trials. The system combines AHRQ costs and FAERS data to estimate downstream direct medical costs. The 2 highest individual scores were attributed to thalidomide analogues, while 14 of the top 50 highest scores were attributable to kinase inhibitors. In general, EPC drug classes with the highest average scores were kinase inhibitor, endothelin receptor antagonist, hepatitis C virus NS3/4A protease inhibitor, recombinant human interferon beta, vascular endothelial growth factor-directed antibody, and tumor necrosis factor blocker. ATC drug class results of note included the findings that antineoplastic drugs were outliers with approximately $80 \%$ of their individual scores at 60 and above, as well as blood and anti-infective drugs with approximately 20\%-30\% of their scores at 60 and above. While some drug classes had a narrow range of scores, certain within-drug class analyses demonstrated broad score ranges for similar medications.

We believe this system provides an accessible reference point regarding real-world differences in safety observed during a drug's postmarketing phase. Because the scoring system is based on direct medical costs, it may be used to improve patient safety by identifying medications that cause undue burdens on patients and health care providers. These scores can be used for identification of drugs that are driving increased costs, as well as assessments and comparisons within a given mechanism of action, across nonrelated drugs, within a drug class, or within a treatment indication. 
Within managed care, the assessment of formulary or preferred status from an individual organizations safety data could be improved by including estimated direct medical costs from AEs and patient outcomes from millions of case reports contained in FAERS. The scores could be used to help health care decision makers drive safer prescribing behavior by optimizing the construction of drug formularies, budget impact models, and by adding an $\mathrm{AE}$ component to population health initiatives. Within-drug class score differences provide head-to-head comparisons that can be utilized for tier placements as well as prior authorization and step-therapy decisions.

\section{Limitations}

A number of constraints must be considered when using and interpreting this system, including limitations regarding reporting rates and potential biases contained in FAERS. Analysis of drug side effects using FAERS has well-recognized limitations. First, since the FDA does not require a causal relationship to exist for an event to be reported, there is no certainty that the reported event was actually due to the reported drug. Second, many of the events that occur are never reported; therefore, the FDA does not recommend using FAERS to estimate incidence rates. ${ }^{1}$ Third, FAERS reporting rates are likely to be low in general and may not be similar across the included drugs or within a given drug class. A detailed analysis of these points was not possible with the data we had access to. Low reporting rates could result in artificially low direct medical cost calculations.

The "primary suspect" designation in FAERS is subjective, and the influence of other drugs or factors cannot be ruled out from a given case report. While we excluded obvious cases where a disease-related symptom was mistakenly denoted as an $\mathrm{AE}$, we assume that we did not catch all such mistakes. Tatonetti et al., Edwards et al. (2005), and Auerbach and Kane (2012) provide more detailed reviews of FAERS limitations. ${ }^{8,51,52}$

Our cost estimates come from mapping AHRQ HCUP cost survey data to MedDRA terms found in FAERS. While we believe this is appropriate, we could not determine if variations between FAERS patient populations and those used for HCUP surveys could influence the results presented here. Additionally, we chose to include only the most expensive AE or outcome in a given case report. Two or more highly related $\mathrm{AE}$ terms are sometimes listed in the same case report, and we believed that including both would artificially inflate the direct medical costs. While this methodological exclusion may be appropriate, it may also have resulted in improper decreases in direct medical cost estimates for certain drugs. Finally, limitations in the accuracy of MEPS data and patient exposure estimates used here may cause artificial increases, or decreases, in calculated direct medical costs.

As with all aspects related to human health, no 1 element should be considered on its own but instead should be viewed as a component in the overall safety picture. FAERS limitations and other qualifications that we noted should always be considered when discussing the results presented here. We vigorously recommend that patients must have consultations with their prescribing physicians before taking any action that relates to FAERS or the analytic system presented in this article.

\section{Conclusions}

Multiple limitations associated with preapproval clinical trials, inadequate data disclosures, slow reaction times from regulatory bodies, and deep-rooted bias against disclosing and publishing negative results drive an acute need for the development of safety analytics that reflect adverse drug effects in heterogeneous, real-world populations. The drug safety analytic detailed here estimates downstream direct medical costs associated with side effects and patient outcomes based on postmarket reports submitted to the FDA. By quantifying the postapproval phase of a drug into downstream direct medical costs, the system can serve as a needed window into the realworld patient population. Future work will hopefully include the integration of additional postmarketing AE databases, such as that of the World Health Organization, as well as electronic health records. ${ }^{8,48}$

\section{Authors}

KEITH B. HOFFMAN, PhD, is Vice President of Scientific Affairs; MO DIMBIL, BS, is Senior Analyst; ROBERT F. KYLE, JD, is Chief Product Officer; COLIN B. ERDMAN, BA, is Technical Lead; ANDREA DEMAKAS, BA, is Product Specialist; DINGGUO CHEN, BS, is Data Engineer; and BRIAN M. OVERSTREET, BA, is Chief Executive Officer, Advera Health Analytics, Santa Rosa, California. NICHOLAS P. TATONETTI, PhD, is Assistant Professor, Department of Biomedical Informatics, Columbia University, New York City, New York.

AUTHOR CORRESPONDENCE: Keith B. Hoffman, PhD, Advera Health Analytics, 3663 N. Laughlin Rd., Ste. 102, Santa Rosa, CA 95403. Tel.: 707.387.9230; Fax: 707.387.9230;

E-mail:keith@adverahealth.com.

\section{DISCLOSURES}

The authors declare no conflicts of interest.

Study concept and design were primarily contributed by Hoffman and Overstreet, along with Kyle and Erdman. Dimbil, Demakas, and Chen had primary responsibility for data collection, with assistance from Erdman. Data interpretation was performed by Hoffman and Tatonetti, with assistance from Kyle, Erdman, and Dimbil. The manuscript was written and revised by Hoffman, with assistance from Dimbil and Kyle. 


\section{ACKNOWLEDGMENTS}

MedDRA, the Medical Dictionary for Regulatory Activities terminology, is the international medical terminology developed under the auspices of the International Conference on Harmonization of Technical Requirements for Registration of Pharmaceuticals for Human Use (ICH).

\section{REFERENCES}

1. Ahmad SR. Adverse drug event monitoring at the Food and Drug Administration. J Gen Intern Med. 2003;18(1):57-60

2. U.S. Food and Drug Administration. Follow-up to the November 2009 early communication about an ongoing safety review of sibutramine, marketed as Meridia. January 21, 2010. Available at: http://www.fda.gov/Drugs/ DrugSafety/PostmarketDrugSafetyInformationforPatientsandProviders/ DrugSafetyInformationforHeathcareProfessionals/ucm 198206.htm. Accessed October 1, 2015

3. Charatan F. Bayer decides to withdraw cholesterol lowering drug. BMJ. 2001;323(7309):359.

4. U.S. Food and Drug Administration. Safety information. Vioxx (rofecoxib) May 2002. Available at: http://www.fda.gov/Safety/MedWatch/ SafetyInformation/SafetyAlertsforHumanMedicalProducts/ucm 154520.htm. Accessed October 1, 2015.

5. Gruber S, van der Laan MJ. An application of targeted maximum likelihood estimation to the meta-analysis of safety data. Biometrics. 2013;69(1):254-62.

6. Cole LW, Kesselheim JC, Kesselheim AS. Ethical issues in new drug prescribing. J Bioeth Inq. 2012;9(1):77-83.

7. U.S. Food and Drug Administration. Reports received and reports entered into FAERS by year. As of December 31, 2013. Available at: http://www. fda.gov/Drugs/GuidanceComplianceRegulatoryInformation/Surveillance/ AdverseDrugEffects/ucm070434.htm. Accessed October 1, 2015.

8. Tatonetti NP, Ye PP, Daneshjou R, Altman RB. Data-driven prediction of drug effects and interactions. Sci Transl Med. 2012;4(125):125ra31.

9. Harpaz R, DuMouchel W, Shah NH, Madigan D, Ryan P, Friedman C. Novel data-mining methodologies for adverse drug event discovery and analysis. Clin Pharmacol Ther. 2012;91(6):1010-21.

10. U.S. Food and Drug Administration. FDA Adverse Event Reporting System (FAERS). 2012. Page updated September 8, 2014. Available at: http:// www.fda.gov/drugs/guidancecomplianceregulatoryinformation/surveillance/ adversedrugeffects/default.htm. Accessed October 1, 2015.

11. Szarfman A, Tonning JM, Doraiswamy PM. Pharmacovigilance in the 21st century: new systematic tools for an old problem. Pharmacotherapy. 2004:24(9):1099-104.

12. Poluzzi E, Raschi E, Koci A, et al. Antipsychotics and torsadogenic risk: signals emerging from the U.S. FDA Adverse Event Reporting System Database. Drug Saf. 2013;36(6):467-79.

13. Hochberg AM, Hauben M. Time-to-signal comparison for drug safety data-mining algorithms vs. traditional signaling criteria. Clin Pharmacol Ther. 2009;85(6):600-06.

14. Robertson HT, Allison DB. Drugs associated with more suicidal ideations are also associated with more suicide attempts. PloS One. 2009;4(10):e7312.

15. Weaver J, Grenade LL, Kwon H, Avigan M. Finding, evaluating, and managing drug-related risks: approaches taken by the US Food and Drug Administration (FDA). Dermatol Ther. 2009;22(3):204-15.

16. Bailey S, Singh A, Azadian R, Huber P, Blum M. Prospective data mining of six products in the US FDA Adverse Event Reporting System: disposition of events identified and impact on product safety profiles. Drug Saf.

2010;33(2):139-46
17. Harpaz R, Chase HS, Friedman C. Mining multi-item drug adverse effect associations in spontaneous reporting systems. BMC Bioinformatics. 2010;11(Suppl 9):S7.

18. Moore TJ, Glenmullen J, Furberg CD. Prescription drugs associated with reports of violence towards others. PloS One. 2010;5(12):e15337.

19. Wang HW, Hochberg AM, Pearson RK, Hauben M. An experimental investigation of masking in the US FDA adverse event reporting system database. Drug Saf. 2010;33(12):1117-33.

20. Moore TJ, Furberg CD, Glenmullen J, Maltsberger JT, Singh S. Suicidal behavior and depression in smoking cessation treatments. PloS One. 2011;6(11):e27016.

21. Sakaeda T, Kadoyama K, Okuno Y. Statin-associated muscular and renal adverse events: data mining of the public version of the FDA adverse event reporting system. PloS One. 2011;6(12):e28124.

22. Takarabe M, Kotera M, Nishimura Y, Goto S, Yamanishi Y. Drug target prediction using adverse event report systems: a pharmacogenomic approach. Bioinformatics. 2012;28(18):i611-i618.

23. Tamura T, Sakaeda T, Kadoyama K, Okuno Y. Aspirin- and clopidogrelassociated bleeding complications: data mining of the public version of the FDA adverse event reporting system, AERS. Int J Med Sci. 2012;9(6):441-46.

24. Chen HC, Tsong Y, Chen JJ. Data mining for signal detection of adverse event safety data. J Biopharm Stat. 2013;23(1):146-60.

25. Harpaz R, DuMouchel W, LePendu P, Bauer-Mehren A, Ryan P, Shah NH. Performance of pharmacovigilance signal-detection algorithms for the FDA adverse event reporting system. Clin Pharmacol Ther. 2013;93(6):539-46.

26. Agency for Healthcare Research and Quality. Healthcare Cost and Utilization Project (HCUP). April 2015. Available at: http://www.ahrq.gov/ research/data/hcup/index.html. Accessed October 1, 2015.

27. Healthcare Cost and Utilization Project (HCUP). Welcome to HCUPnet. Agency for Healthcare Research and Quality. Available at: http://hcupnet. ahrq.gov/HCUPnet.jsp. Accessed October 1, 2015.

28. Hoffman KB, Overstreet BM, Doraiswamy PM. A drug safety ePlatform for physicians, pharmacists and consumers based on post-marketing adverse events. Drugs and Therapy Studies. 2013;3(1):e4.

29. Medical Dictionary for Regulatory Activities (MedDRA). 2015. Available at: http://www.meddra.org/. Accessed October 14, 2015.

30. National Library of Medicine. RxNorm. Page updated December 1, 2014. Available at: http://www.nlm.nih.gov/research/umls/rxnorm/. Accessed October 1, 2015.

31. U.S. Food and Drug Administration. Pharmacologic Class: National Drug File reference terminology. 2013. Page updated August 18, 2015. Available at: http://www.fda.gov/ForIndustry/DataStandards/ StructuredProductLabeling/ucm 162549.htm. Accessed October 1, 2015.

32. U.S. Food and Drug Administration. Structured product labeling resources. 2015. Available at: http://www.fda.gov/ForIndustry/ Data Standards/StructuredProductLabeling/default.htm. Accessed October 14, 2015

33. National Center for Biomedical Ontology. BioPortal mappings. Available at: http://bioportal.bioontology.org/mappings. Accessed October 13, 2015.

34. ICD9Data. com. The Web's free 2015 medical coding reference. 2015. Available at: http://www.icd9data.com/. Accessed October 1, 2015.

35. Healthcare Cost and Utilization Project (HCUP). HCUP statistical briefs chronological. Agency for Healthcare Research and Quality. September 2015. Rockville, MD. Available at: http://www.hcup-us.ahrq.gov/reports/statbriefs/statbriefs.jsp. Accessed October 1, 2015.

36. EudraVigilance. Expert Working Group: Important Medical Event Terms (IME) list. 2013. Page updated October 1, 2015. Available at: http://eudravigilance.ema.europa.eu/human/textforIME.asp. Accessed October 1, 2015. 
37. Agency for Healthcare Research and Quality. Medical Expenditure Panel Survey (MEPS). Page updated August 21, 2009. Available at: http://meps. ahrq.gov/mepsweb/about_meps/survey_back.jsp. Accessed October 1, 2015.

38. WHO Collaborating Centre for Drug Statistics Methodology. Guidelines for ATC classification and DDD assignment. 2013. Page updated May 5, 2015. Available at: http://www.whocc.no. Accessed October 1, 2015.

39. Hemminki E. Study of information submitted by drug companies to licensing authorities. Br Med J. 1980;280(6217):833-36.

40. Cowley AJ, Skene A, Stainer K, Hampton JR. The effect of lorcainide on arrhythmias and survival in patients with acute myocardial infarction: an example of publication bias. Int J Cardiol. 1993;40(2):161-66.

41. Lundh A, Sismondo S, Lexchin J, Busuioc OA, Bero L. Industry sponsorship and research outcome. Cochrane Database Syst Rev. 2012;12:MR000033.

42. Bero L. Industry sponsorship and research outcome: a Cochrane review. JAMA Intern Med. 2013;173(7):580-81.

43. Lexchin J, Bero LA, Djulbegovic B, Clark O. Pharmaceutical industry sponsorship and research outcome and quality: systematic review. BMJ. 2003;326(7400):1167-70.

44. Melander H, Ahlqvist-Rastad J, Meijer G, Beermann B. Evidence b(i)ased medicine-selective reporting from studies sponsored by pharmaceutical industry: review of studies in new drug applications. BMJ. 2003;326(7400):1171-73.
45. Eyding D, Lelgemann M, Grouven U, et al. Reboxetine for acute treatment of major depression: systematic review and meta-analysis of published and unpublished placebo and selective serotonin reuptake inhibitor controlled trials. BMJ. 2010;341:c4737.

46. Chan AW, Hrobjartsson A, Haahr MT, Gotzsche PC, Altman DG Empirical evidence for selective reporting of outcomes in randomized trials: comparison of protocols to published articles. JAMA. 2004;291(20):2457-65.

47. Ernst \& Young. Progressions: Navigating the payer landscape. Global Pharmaceutical Report 2014. Available at: http://www.ey.com/ Publication/vwLUAssets/EY_-_Progressions_2014:_Navigating_the_payer_ landscape/\$FILE/EY-progressions-2014.pdf. Accessed October 1, 2015.

48. Harpaz R, Vilar S, Dumouchel W, et al. Combing signals from spontaneous reports and electronic health records for detection of adverse drug reactions. J Am Med Inform Assoc. 2013;20(3):413-19.

49. Poluzzi E, Raschi E, Moretti U, De Ponti F. Drug-induced torsades de pointes: data mining of the public version of the FDA Adverse Event Reporting System (AERS). Pharmacoepidemiol Drug Saf. 2009;18(6):512-18.

50. Wilson AM, Thabane L, Holbrook A. Application of data mining techniques in pharmacovigilance. Br J Clin Pharmacol. 2004;57(2):127-34.

51. Edwards R, Faich G, Tilson H. Points to consider: the roles of surveillance and epidemiology in advancing drug safety. Pharmacoepidemiol Drug Saf. 2005;14(9):665-67.

52. Auerbach M, Kane RC. Caution in making inferences from FDA's Adverse Event Reporting System. Am J Health Syst Pharm. 2012;69(11):922-23. 


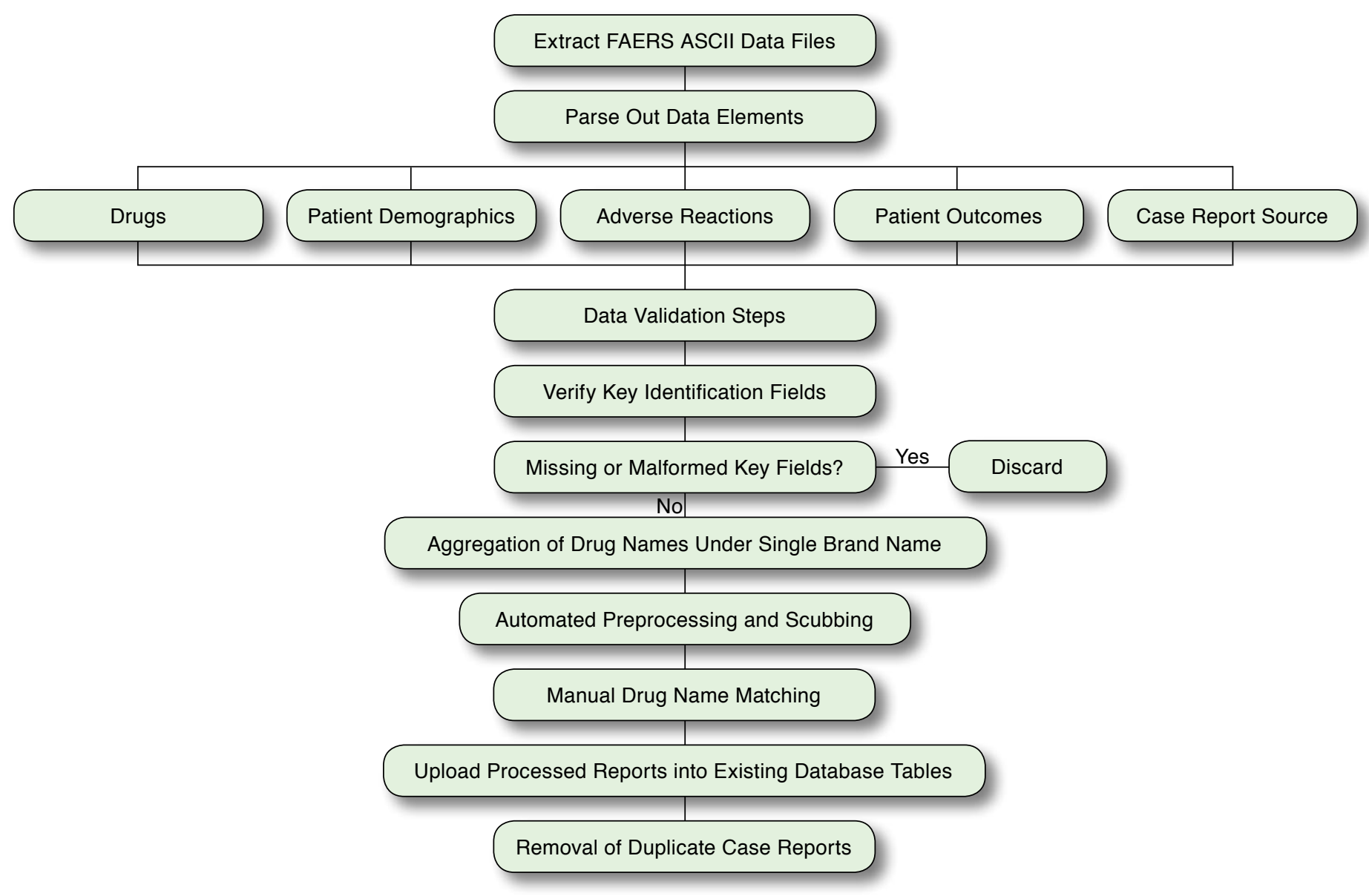

FAERS = FDA Adverse Event Reporting System database.

APPENDIX B Examples of HCUP Survey Costs Mapped to ICD-9-CM and MedDRA Terms

\begin{tabular}{|c|c|c|c|c|}
\hline MedDRA Preferred Term & ICD-9-CM & $\begin{array}{l}\text { Number of } \\
\text { Discharges }\end{array}$ & $\begin{array}{l}\text { Aggregate Direct } \\
\text { Medical Costs (\$) }\end{array}$ & $\begin{array}{c}\text { Mean Costs } \\
(\$)\end{array}$ \\
\hline Arterial rupture & Rupture of artery (447.2) & 385 & $14,391,896$ & 37,382 \\
\hline Intestinal perforation & Perforation of intestine (569.83) & 8,600 & $265,711,974$ & 30,897 \\
\hline Progressive multifocal leukoencephalopathy & Progressive multifocal leukoencephalopathy (046.3) & 260 & $5,881,891$ & 22,623 \\
\hline Neutropenia & Neutropenia, unspecified (288.00) & 42,500 & $513,536,684$ & 12,083 \\
\hline Stevens-Johnson syndrome & Stevens-Johnson syndrome (695.13) & 2,235 & $25,062,983$ & 11,214 \\
\hline Atrial fibrillation & Atrial fibrillation (427.31) & 438,025 & $3,706,927,755$ & 8,463 \\
\hline Rhabdomyolysis & Rhabdomyolysis (728.88) & 38,310 & $288,778,860$ & 7,538 \\
\hline Renal failure & Renal failure, unspecified (586) & 990 & $7,120,651$ & 7,193 \\
\hline Urinary tract infection & Urinary tract infection, site not specified (599.0) & 416,935 & $2,758,725,997$ & 6,617 \\
\hline Loss of consciousness & Syncope and collapse (780.2) & 198,260 & $1,212,736,491$ & 6,117 \\
\hline
\end{tabular}

HCUP = Healthcare Cost and Utilization Project; ICD-9-CM = International Classification of Diseases, Ninth Revision, Clinical Modification; MedDRA= Medical Dictionary for Regulatory Activities. 
APPENDIX C Distribution of Weighted Average Scores for EPCs Comprising 3 or More Individual Compounds and 3,000 or More Case Reports

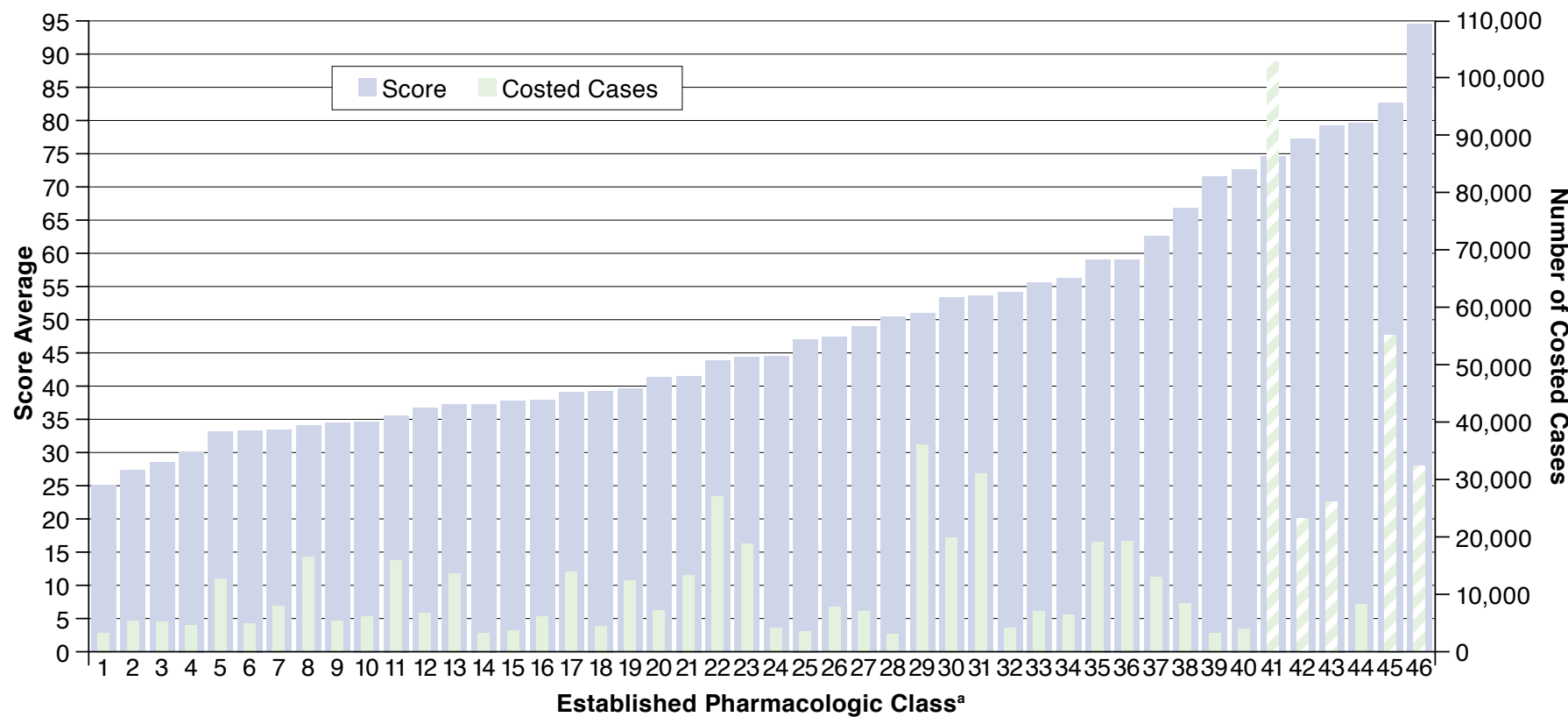

\begin{tabular}{|c|c|c|c|}
\hline \# & EPC Legend & \# & EPC Legend \\
\hline 1 & Penicillin-class antibacterial & 24 & Antiarrhythmic \\
\hline 2 & Angiotensin-converting enzyme inhibitor & 25 & Cholinesterase inhibitor \\
\hline 3 & beta-adrenergic blocker & 26 & Mood stabilizer \\
\hline 4 & Thiazide diuretic & 27 & Dipeptidyl peptidase 4 inhibitor \\
\hline 5 & Corticosteroid & 28 & Phosphodiesterase 5 inhibitor \\
\hline 6 & Dihydropyridine calcium channel blocker & 29 & Progestin \\
\hline 7 & beta2-adrenergic agonist & 30 & Bisphosphonate \\
\hline 8 & HMG-CoA reductase inhibitor & 31 & Atypical antipsychotic \\
\hline 9 & Biguanide & 32 & $\begin{array}{l}\text { HIV nucleoside analog reverse } \\
\text { transcriptase inhibitor }\end{array}$ \\
\hline 10 & Benzodiazepine & 33 & $\begin{array}{l}\text { transcriptase inhibitor } \\
\text { GLP-1 receptor agonist }\end{array}$ \\
\hline 11 & Opioid agonist & 34 & Calcineurin inhibitor immunosuppressant \\
\hline 12 & Nonsteroidal anti-inflammatory drug & $\frac{34}{35}$ & Thiazolidinedione \\
\hline 13 & Proton pump inhibitor & 36 & Peroxisome proliferator receptor gamma agonist \\
\hline 14 & Peroxisome proliferator receptor alpha agonist & 37 & Factor Xa inhibitor \\
\hline 15 & gamma-aminobutyric acid-ergic agonist & 38 & Nucleoside metabolic inhibitor \\
\hline 16 & Quinolone antimicrobial & 39 & Microtubule inhibitor \\
\hline 17 & Serotonin-reuptake inhibitor & 40 & Alkylating drug \\
\hline 18 & Anticholinergic & 41 & Tumor necrosis factor blocker \\
\hline 19 & Angiotensin 2 receptor blocker & 42 & Vascular endothelial growth factor-directed antibody \\
\hline 20 & Serotonin and norepinephrine reuptake inhibitor & 43 & Recombinant human interferon beta \\
\hline 21 & Insulin analog & 44 & Hepatitis C virus NS3/4A protease inhibitor \\
\hline 22 & Anti-epileptic agent & 45 & Kinase inhibitor \\
\hline 23 & Estrogen & 46 & Endothelin receptor antagonist \\
\hline
\end{tabular}


APPENDIX D Percentage of Scores of 60 or More for Each ATC Group with 10 or More Individual Drugs

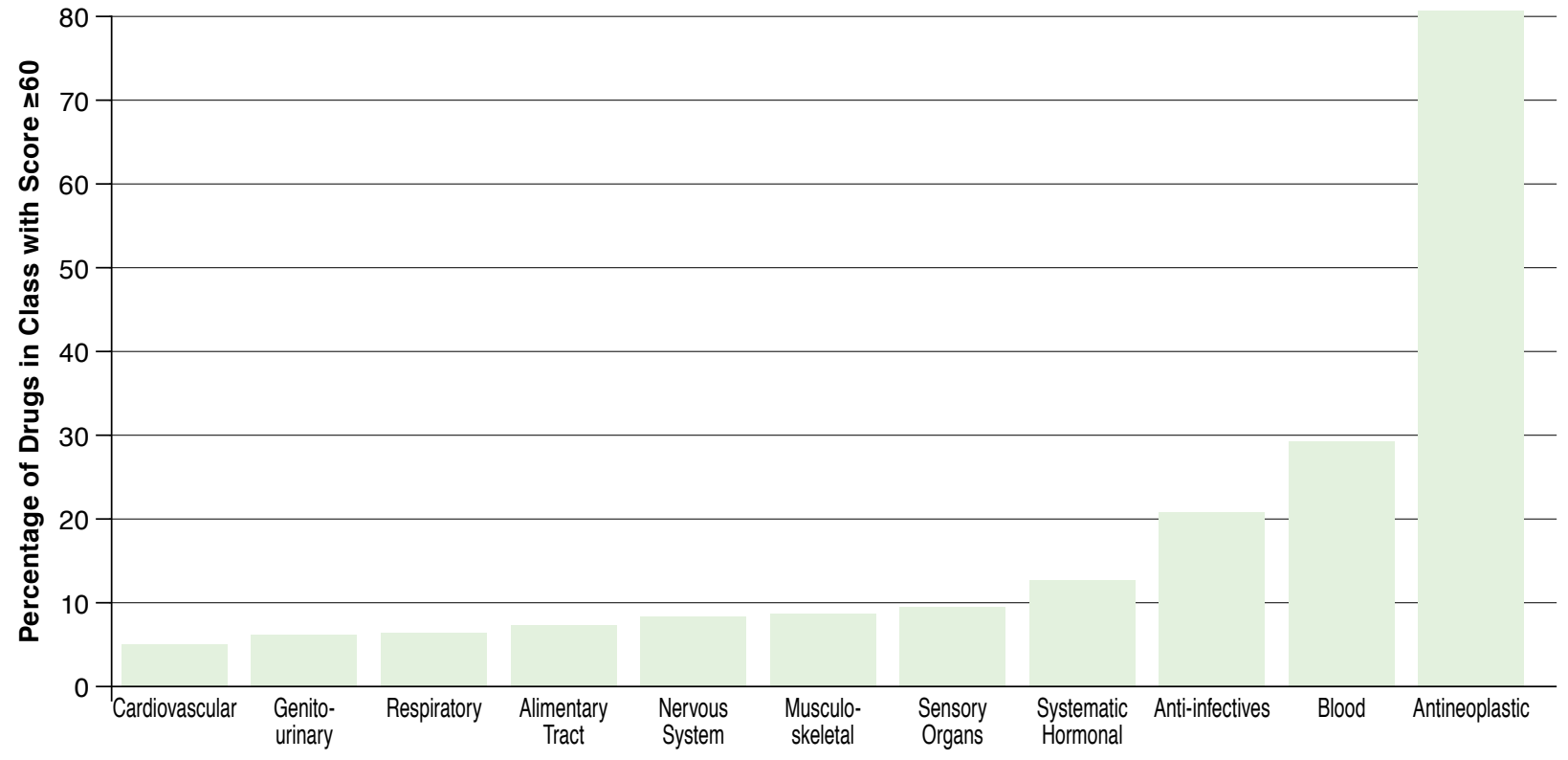

ATC=Anatomical Therapeutic Chemical. 